\title{
Amplified spontaneous emission spectroscopy on semiconductor optical amplifiers subject to active light injection
}

\author{
A. P. de Boer, ${ }^{\text {a) }}$ P. C. M. Christianen, J. C. Maan, and Th. Rasing \\ Research Institute for Materials and High Field Magnet Laboratory, University of Nijmegen, \\ Toernooiveld 1, 6525 ED Nijmegen, The Netherlands \\ V. I. Tolstikhin and T. G. van de Roer \\ Department of Electrical Engineering, Eindhoven University of Technology, $5600 \mathrm{MB}$ Eindhoven, \\ The Netherlands \\ H. M. de Vrieze \\ Philips Optoelectronics Research, Prof. Holstlaan 4, 5656 AA Eindhoven, The Netherlands
}

(Received 23 October 1997; accepted for publication 7 April 1998)

\begin{abstract}
It is shown that measurements of the effect of optical injection with an external laser on the spectral response of a semiconductor optical amplifier can probe intrinsic properties of a working device. The data demonstrate that under saturated gain conditions the carrier energy distribution within the active layer of a AlGaAs/GaAs amplifier neither shows spectral hole burning nor carrier heating, but only a decreased density. (C) 1998 American Institute of Physics. [S0003-6951(98)02823-X]
\end{abstract}

Knowledge of the response of the carrier distribution to the strong internal optical fields in semiconductor lasers and semiconductor optical amplifiers is essential for optimizing the performance of these devices. Usually this information is inferred from spontaneous emission spectra. ${ }^{1-3}$ However, in working lasers spontaneous emission spectra suffer from the presence of a longitudinal mode pattern, which complicates the interpretation and hampers a reliable determination of properties from these spectra.

In this letter we present a novel method to study the properties of inverted semiconductor devices. A key point in our approach is that by injecting an external laser beam into the waveguide of a semiconductor optical amplifier (SOA), changes in the carrier energy distribution are induced which are similar to those in real laser devices due to the intracavity optical fields. Since an SOA has anti-reflectivity (AR) coated facets, the formation of longitudinal Fabry-Pérot modes in the waveguide is avoided. Hence, by measuring the full spectral response of the amplified spontaneous emission (ASE) that is emitted from the edge of the device, an undisturbed fingerprint of the carrier distribution is obtained. Our method can be regarded as a model experiment for device studies under real laser operation. By tuning the external laser one has full control over the intensity, polarization, and wavelength of the laser optical field, independent of the biasing conditions. Furthermore real devices, without a special need for invasive processing to create an optical access perpendicular to the waveguide, can be used. This is in particular advantageous, because such an optical access can also give rise to Fabry-Pérot-like oscillatory features in spontaneous emission spectra. ${ }^{2,3}$ Finally the technique can be extended to dynamic studies with sub-ps time resolution using an upconversion scheme.

The device under study is a GaAs/AlGaAs double heterostructure ridge waveguide laser diode with AR-coated

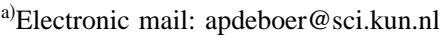

facets, fabricated at Philips Optoelectronics Research. The ridge is etched at $4^{\circ}$ to the normal of the cleavage planes. The $40 \mathrm{~nm}$ thick $\mathrm{Al}_{0.13} \mathrm{Ga}_{0.87} \mathrm{As}$ active layer is embedded in $1.5 \mu \mathrm{m}$ thick $\mathrm{Al}_{0.5} \mathrm{Ga}_{0.5} \mathrm{As}$ cladding layers. The SOA is operated with a pulsed current source with a pulse width of about $10 \mu$ s and a duty cycle of $3 \%$ to reduce dissipation. A continuous wave Ti:sapphire laser, tunable from 700 to 860 $\mathrm{nm}$, is coupled into the front facet of the waveguide with a microscope objective. The ASE is collected at the end facet of the waveguide with a similar microscope objective and guided into a grating monochromator system. For accurate comparison ASE spectra with and without the injected laser field are measured quasi-simultaneously. For this purpose a mechanical chopper, synchronized with the current source and a gated photon counting system, is used to chop the injected laser beam. In the dark period one measures the steady state ASE spectrum and in the light period the injected laser induced change in the ASE spectral distribution is measured.

Experimentally obtained ASE spectra for different wavelengths of the external laser are plotted in Fig. 1. For all spectra the SOA is biased at $60 \mathrm{~mA}$ to have a substantial inversion and gain. The single pass peak gain is approximately $12 \mathrm{~dB}$. The wavelength of the external laser is tuned within the gain regime to induce strong stimulated emission. Injecting the external laser into the wavelength leads to a strong decrease of the ASE spectral intensity over its whole energy range (Fig. 1). Optical injection creates an additional recombination channel that competes with the nonradiative channel and the ASE recombination channel for carriers, effectively reducing the inversion population density.

To illustrate the use of our technique the gain profile in the device is determined by tuning the wavelength of the external laser. In Fig. 2, it is shown that as a function of the input wavelength, the output intensity of the SOA [Fig. 2(b)] exhibits a behavior similar to the relative change of the integrated ASE spectral intensity after removing the stimulated emission spike [Fig. 2(a)]. Also the effect of the optical con- 


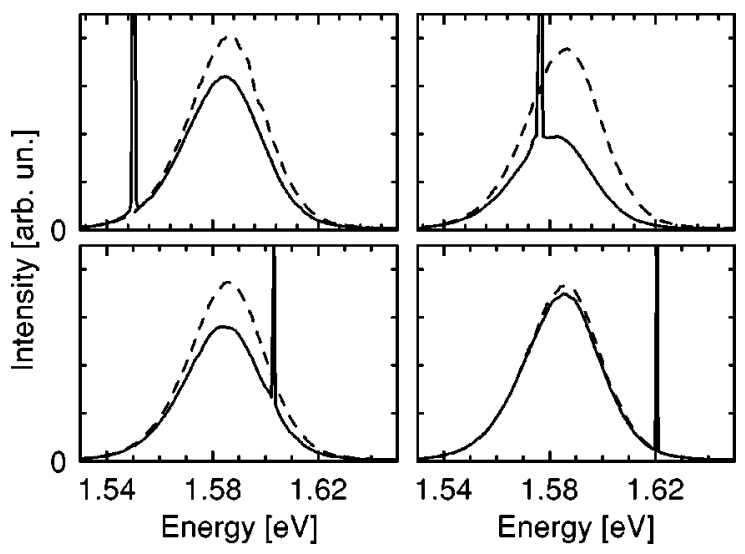

FIG. 1. ASE spectra with (solid line) and without (dashed line) optical injection under the same biasing conditions plotted on a linear scale. The stimulated emission spike corresponds to the energy of the external laser.

finement factor, which accounts for the intensity overlap of the laser field with the active layer, is clearly demonstrated. For the transverse magnetic (TM) polarization the response is smaller than for the transverse electric (TE) polarization. Since the variations in the coupling efficiency are kept mini$\mathrm{mal}$, the measured difference is due mainly to the optical confinement factor, which for TM polarized light is about $25 \%$ smaller than for TE polarized light.

More quantitatively these results can be described with the carrier density rate equation ${ }^{4}$

$$
\frac{d N}{d t}=\frac{i_{\text {bias }}}{e V}-\gamma_{\mathrm{nr}} N-S_{\text {ase }}(N)-\frac{\Gamma G_{L} I_{L}}{\hbar \omega_{L}}
$$

where $N$ is the carrier density, $i_{\text {bias }}$ the bias current, $e$ the electron charge, $V$ the waveguide volume, $\gamma_{\mathrm{nr}}$ the nonradiative recombination rate, $S_{\text {ase }}(N)$ the ASE emission rate, $\Gamma$ the optical confinement factor, $G_{L}=G_{L}\left(\hbar \omega_{L}\right)$ the gain at the laser energy, $I_{L}$ the laser intensity, and $\hbar \omega_{L}$ the laser photon energy. The last term in (1) represents the rate of stimulated emission induced by the injection of the external laser beam. Since the bias current remains the same, it follows directly from (1) that the presence of stimulated emission leads to a reduced carrier density $N_{L}$. As a consequence, the ASE emission rate decreases. Using (1), this external laser induced change in $S_{\text {ase }}$ can in the steady state $[(d N / d t)=0]$ be expressed as

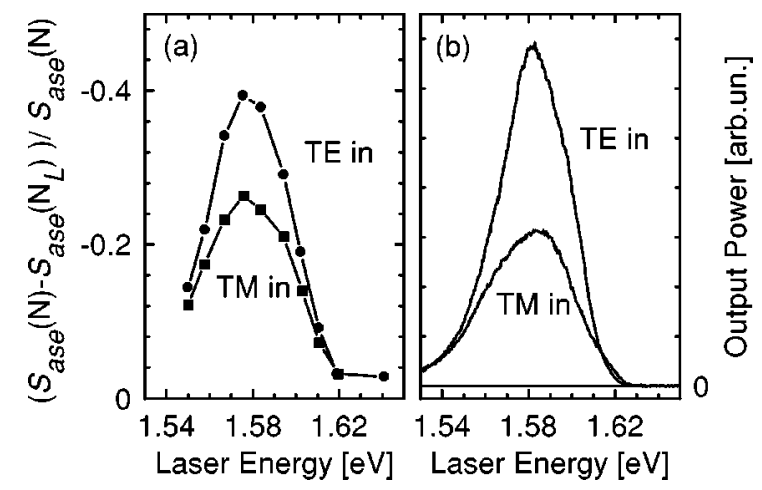

FIG. 2. Relative change of the integrated ASE intensity (a) and output

intensity (b) vs energy of the external laser.
Downloaded 16 Jun 2008 to 131.174.20.161. Redistribution subject to AlP license or copyright; see http://apl.aip.org/apl/copyright.jsp

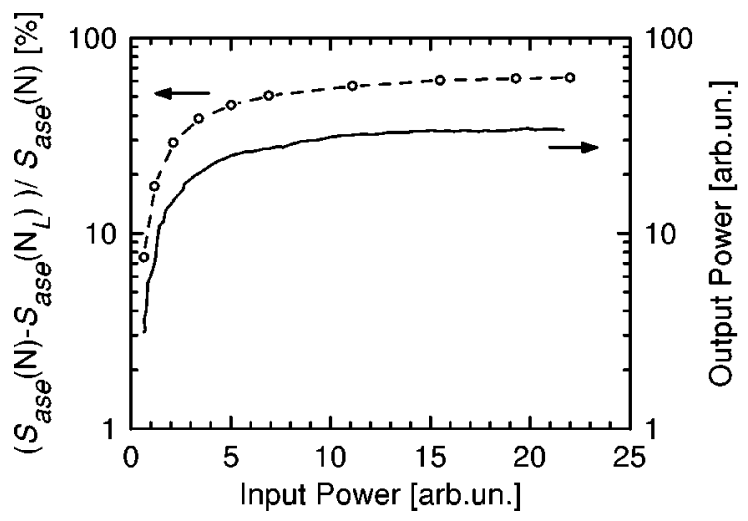

FIG. 3. Output power (solid line) and relative change of the integrated ASE intensity (dashed line) vs input power at a bias current level of $88 \mathrm{~mA}$.

$$
S_{\text {ase }}(N)-S_{\text {ase }}\left(N_{L}\right)=\frac{\Gamma G_{L} I_{L}}{\hbar \omega_{L}}-\gamma_{\mathrm{nr}}\left(N-N_{L}\right) .
$$

Although this representation does not take the actual energy distribution of the carriers into account, it adequately explains the observed features so far. In any case, by resolving the response of the ASE intensity in the spectral domain our technique probes stimulated emission induced effects that go beyond this description as well. Since in the present case the stimulated emission is the adjustable parameter, the dependence on gain, polarization, and laser intensity can be investigated. By measuring close to the saturation of the amplifier it is justified to ignore longitudinal features in our model, because saturation assures longitudinal homogeneity.

To understand the saturation behavior of the SOA we measured the spectral response of the ASE to injection with a high intensity laser beam. Besides a linear dependence of the gain on the population inversion density, nonlinear processes-like carrier heating and spectral hole burning (SHB) - are considered to be important for gain saturation in semiconductor lasers and amplifiers. ${ }^{5-7}$ Carrier heating arises because in the stimulated recombination process carriers with an energy less than the average energy are involved. Removing these carriers from the distribution increases the average energy of the remaining carriers, leading to a distribution with a higher effective temperature. Spectral hole burning is seen as a deviation of the carrier distribution from the Fermi-Dirac distribution due to stimulated recombination at one particular energy. Both processes can give rise to nonlinear gain saturation as was claimed in previous publications. ${ }^{5-7}$ However, those experiments were not carried out in the spectral domain.

In Fig. 3 the output power and the relative change of the integrated ASE intensity is plotted as a function of the input power of the external laser. The saturation of the output power coincides exactly with the saturation of the relative change of the integrated ASE spectral intensity. This result suggests that the gain in a bulk semiconductor scales only linearly with the carrier density minus the transparency carrier density, ${ }^{4}$ meaning that with increasing input intensity the depletion of the carrier distribution proceeds until the transparency condition for the incident laser photon energy is fulfilled.

To examine the nonlinear contributions to the gain saturation, we measured the spectral response in the highly satu- 


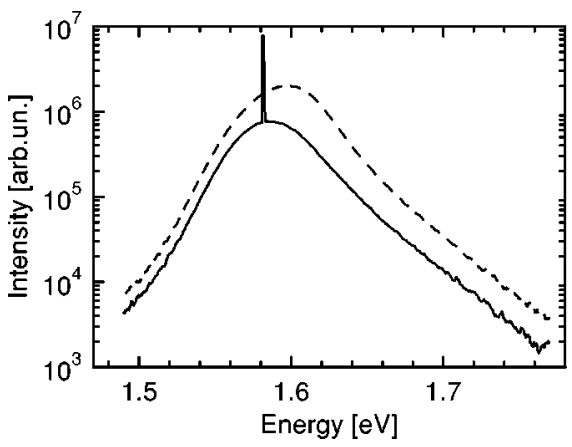

FIG. 4. ASE spectra without (dashed line) and with (solid line) injecting an intense saturating laser beam into the SOA at a bias current level of $88 \mathrm{~mA}$ plotted on a semi-logarithmic scale showing that the high energy tail (三effective temperature) remains unchanged.

rated regime over more than 3 decades. From plotting ASE spectra with and without injection of the intense laser beam on a semi-logarithmic scale (Fig. 4), it can be seen that the achieved saturation results in a smooth and strong decrease of the ASE intensity over its whole energy range. Furthermore, the high energy tails of both spectra have the same slope even under these highly saturated conditions. The experiment shows that relaxation of carriers via carrier-carrier scattering and the cooling of the carrier distribution via inelastic phonon scattering take place at such high rates that the carriers relax to a distribution with a lower density, which has neither a persisting spectral hole nor an altered effective carrier temperature. Thus nonlinear gain saturation arising from $S H B$ or carrier heating is not present in the amplifier. This contrasts with earlier work obtained on laser diodes with an optical access perpendicular to the waveguide. ${ }^{2}$ In that work the occurrence of both SHB and carrier heating was claimed by comparing spontaneous emission spectra measured at different bias current levels, i.e., at different levels of gain saturation. Our data taken on a SOA do not show any significant influence of SHB or carrier heating. Since SHB is only feasible in the case of a very intense laser field, we estimated the intensity of the optical field inside the waveguide in the saturated regime. Without optical injection a reduction of the drive current with $30 \mathrm{~mA}$ leads to an ASE spectrum with a shape and peak intensity similar to the highly saturated ASE spectrum. This change in drive current $\Delta i$ should equal the stimulated recombination current. Using Eq. (1) the following relation holds:

$$
\frac{\Delta i}{e V}=\frac{\Gamma G_{L} I_{L}}{\hbar \omega_{L}}
$$

from which the intensity inside the waveguide is estimated to be $0.75 \mathrm{MW} / \mathrm{cm}^{2}$. This is far below the intensity needed for $\mathrm{SHB},{ }^{4}$ but sufficient to saturate the gain.

In conclusion, we have demonstrated a new method to characterize semiconductor optical amplifiers which is based on the study of the spectral response of ASE to the internal optical fields, generated by a tunable external laser. We have shown that after saturating the SOA with intense laser power, the carriers redistribute to a lower density with an unchanged effective carrier temperature. Therefore, nonlinear gain saturation due to spectral hole burning or carrier heating is not observed in this device.

The authors would like to thank A. F. van Etteger for excellent technical support, $\mathrm{H}$. Ambrosius for growing the structure, and B. Smalbrugge for mounting the devices. This work was supported by the Stichting Technische Wetenschappen (STW) with financial support from the Nederlandse Organisatie voor Wetenschappelijk Onderzoek (NWO).

${ }^{1}$ C. H. Henry, R. A. Logan, and F. R. Merritt, J. Appl. Phys. 51, 3042 (1980).

${ }^{2}$ F. Girardin, G. H. Duan, P. Gallion, A. Talneau, and A. Ougazzaden, Appl. Phys. Lett. 67, 771 (1995).

${ }^{3}$ E. H. Sargent, D. Pavlidis, H. Anis, N. Golinescu, J. M. Xu, R. Clayton, and H. B. Kim, J. Appl. Phys. 80, 1904 (1996).

${ }^{4}$ W. W. Chow, S. W. Koch, and M. Sargent III, Semiconductor-Laser Physics (Springer, Berlin, Heidelberg, 1994), Ch. 3.

${ }^{5}$ K. L. Hall, G. Lenz, E. P. Ippen, U. Koren, and G. Raybon, Appl. Phys. Lett. 61, 2512 (1992).

${ }^{6}$ C. K. Sun, H. K. Choi, C. A. Wang, and J. G. Fujimoto, Appl. Phys. Lett. 63, 96 (1993).

${ }^{7}$ J. Zhou, N. Park, J. W. Dawson, K. J. Vahala, M. A. Newkirk, U. Koren, and B. I. Miller, Appl. Phys. Lett. 62, 2301 (1993). 